\title{
Synthesis of Gold-Palladium Bimetallic Nanoparticles and Surface Catalytic Activity in Suzuki Coupling Reactions Using in FTIR Spectroscopy
}

\author{
P. Venkatesan, J. Santhanalakshmi* \\ Department of Physical Chemistry, University of Madras, Guindy Campus, A.C.Tech., Chennai Tamil Nadu, 600025, India
}

\begin{abstract}
Colloidal bimetallic gold core palladium shell nanoparticles were prepared by wet chemical method, in which $\mathrm{Au}(\mathrm{III})$ and $\mathrm{Pd}(\mathrm{II})$ ions in an aqueous solution in the presence of a cationic surfactant, Cetyltrimethylammonium bromide (CTAB). The structure and composition of the metallic nanoparticles were characterized by UV-Vis, HRTEM, SEM-EDX, XRD, XPS and FTIR. The catalytic activities of nanoparticles are tested on the surface chemical characterization study of Cetyltrimethylammonium bromide supported Au-Pd bimetallic nanoparticle catalyst, hereafter named Au-Pdnp, is reported. Such a catalyst was developed for the Suzuki coupling reaction and found excellent catalytic activity. Here we describe the catalytic performance and the FTIR studies provide proof of the mode of binding that occurs in the Palladium nanoparticle surface for the first time and also confirms the mechanism of the Suzuki reaction.
\end{abstract}

Keywords Bimetallic Nanoparticle, Suzuki Coupling Reaction, Surface Study, Thermal Method, FT-IR Study

\section{Introduction}

Nanoparticles exhibit significantly different properties relative to those of their corresponding bulk materials and, as such, are of interest for applications in catalysis[1 - 8], magnetic[9 - 11], drug delivery systems[12, 13], semiconductors[14, 15], single electron tunnelingdevices [16], nonlinear optical devices[17] , electron microscopy markers[18] and DNA sequencing[19]. Of late, bimetallic nanoparticles are of great interest because of the modification of properties observed not only due to size effects, but also as a result of the combination of different metals, either as an alloy or as a core-shell structure, modifying the catalytic properties of the monometallic nanoparticles[20 - 26]. Recently Palladium-catalyzed Suzuki cross-coupling reaction has emerged as one of the most powerful, attractive, and widely utilized method for the construction of carbon-carbon bonds[27-30]. In recent years, there has been considerable interest in the preparation of new and highly active palladium catalysts to facilitate such transformation[31-37]. Water as solvent in transition-metal catalysis has many advantages for the recycling of catalyst, product recovery and environmental aspects[38]. The beneficial effects of using water as solvent especially in

* Corresponding author:

jslakshmi@yahoo.co.in (J. Santhanalakshmi)

Published online at http://journal.sapub.org/materials

Copyright (C) 2012 Scientific \& Academic Publishing. All Rights Reserved
Suzuki reactions are well documented[39,40]. In the present paper a surface chemical characterization study ofCetyltrim ethyl ammonium bromide supported Au-Pd bimetallic nanoparticle catalyst, hereafter named Au-Pdnp, is reported. Such a catalyst was developed for the Suzuki coupling reaction and found excellent catalytic activity. Here we describe the catalytic performance and the FTIR studies provide proof of the mode of binding that occurs in the Palladium nanoparticle surface for the first time and also confirms the mechanism of the Suzuki reaction.

\section{Experimental}

\subsection{Preparation of Au-Pd Bimetallic Nanoparticles}

The bimetallic nanoparticles were synthesized by chemical method reduction of the $\mathrm{Au}^{3+}$ and $\mathrm{Pd}^{2+}$ ions (metal salts) using sodium borohydride $\left(\mathrm{NaBH}_{4}\right)$ as the reducing agent by our group previously established methods[41] The resulting colloidal nanoparticle solutions was very stable over extended periods of several months.

\subsection{Catalytic Study: Suzuki Reaction between Phenyl Halide Derivatives and Phenylboronic Acid to Substituted Biphenyl: General Procedure}

Phenylboronic acid $(1.05 \mathrm{mmol})$, Iodobenzene $(1.0 \mathrm{mmol})$, base $(3 \mathrm{mmol})$ and $0.5 \mathrm{~mol} \%$ catalyst (Au-Pdnp) were introduced under argon atmosphere into a $25-\mathrm{ml}$ round bott omed, two-necked flask equipped with a stirring magnetic 
bar, a bubble condenser, and a silicon stopper. The reaction mixture was magnetically stirred at the required temperature (Table 1). When needed, small samples of the reaction mixture were taken from the stoppered side neck. For GC analysis the samples were treated with either water or $0.5 \mathrm{M}$ aqueous $\mathrm{HCl}$. The organic products were then extracted with diethyl ether, dried over anhydrous sodium sulfate and analyzed by GCMS. The coupling product was purified by column chromatography (silica, solvent: n-hexane/ethyl acetate).

\subsection{FTIR Studies of Dried Films}

FTIR experiments were carried out on a Bruker Tensor 27 spectrophotometer. The spectra were collected in the range 4000 to $400 \mathrm{~cm}^{-1}$ in the transmittance mode. Solid samples were finely ground, dispersed in spectroscopic grade $\mathrm{KBr}$ and made into pellets. Liquid samples prepared in volatile solvents were drop cast onto the $\mathrm{KBr}$ pellets and allowed to dry before recording the IR spectra. A few drops of the solution were placed on a $\mathrm{KBr}$ pellet and allowed to dry before IR spectra were recorded.

\section{Results and Discussion}

\subsection{Investigation of Catalytic Activity of Suzuki Reaction}

The catalytic activity of the bimetallic nanoparticles towards the Suzuki coupling reaction of Phenylboronic acid react with iodobenzene was studied, and the formation of biphenyl were observed. GC results confirmed the presence of biphenyl as a product. In the present work, the synthesis of biphenyl using a catalytic amount of bimetallic nanoparti cles under DMF-water $(3: 1, \mathrm{v} / \mathrm{v})$ solvents conditions. The reaction of Iodobenzene and Phenylboronic acid with Sodium acetate in the presence of catalytic amount of metal nanoparticles at $100{ }^{\circ} \mathrm{C}$ affords the desired biphenyl compounds in $99 \%$ yield (table 1, Sl.no 2). We also examined this reaction in different solvents, base, catalysts and temperature condition (Table 1). The product percentage yields obtained from different reactant compositi ons show the optimum reactant compositions for maximum yield as those mentioned in the Suzuki reaction procedure (table 1). The results of product yields given in table 1 are determined by using the optimum reactant compositions only. The Sl.no 1 and 2 in the table 1 refer to the inferences obtained from the effects of catalysts nature. Among the metallic nanoparticles system, Au-Pd bimetallicnanoparticle catalysis shows maximum yield than mono metallic palladium nanoparticle.

The Sl.no 2, 3 to 5 in the table 1 refer to the results of the effect of the nature of base $(3.0 \mathrm{mmol})$ keeping the reaction temperature $\left(100^{\circ} \mathrm{C}\right)$, solvent (DMF-water $3: 1, \mathrm{v} / \mathrm{v}$ mixture) and 0.5 mole $\%$ catalyst as constant parameters. Maximum product yields for Au-Pd nanoparticle catalysts are found with Sodium acetate as the base and at $100^{\circ} \mathrm{C}$. When Suzuki reaction was carried out with different solvents such as DMF-water and acetonitrile (table 1, Sl. no 2, 6 and 7), the product yields are found lesser for the DMF-water 3:1 mixture as the solvent. The temperature effect studies show that, the optimum reaction temperature is found at $100^{\circ} \mathrm{C}$, since $120^{\circ} \mathrm{C}$ and $150^{\circ} \mathrm{C}$ reaction temperatures also produced the same percentage yield (table 1, Sl. no. 2, 8 and 9). In all, $3.0 \mathrm{mmol}$ Sodium acetate incorporated Suzuki reaction, when carried out in DMF-water (3:1) solvent mixture, loaded with 0.5 mole $\%$ of the catalyst, at $100^{\circ} \mathrm{C}$ produced the maximum percentage yield of the product in the least reaction time $(12 \mathrm{~h})$ when the catalyst is Au-Pd bimetallic nanoparticle. The Sl. no. 2, 10 to 12 in the table 1 refer to the inferences obtained from the effects of catalysts loading to the Suzuki reaction. Among the various concentration of metallic nanoparticles system, Au-Pd nanoparticle with 0.5 and $0.6 \mathrm{~mol} \%$ catalysis shows maximum yield compared to the other nanoparticles system. This reaction studied without catalysts (Sl. no. 13 in the table 1) gave only $36.0 \%$ of the Suzuki products.

Table 1. Reaction conditions, catalysts nature and effects of base, temperature, solvent nature and the product yield of the Suzuki reaction.[Phenylboronic acid] $=1.05 \mathrm{mmol}$,[Iodobenzene] $=1.00 \mathrm{mmol}, 3: 1 \mathrm{molar}$ ratio of DMF-water in thermal method

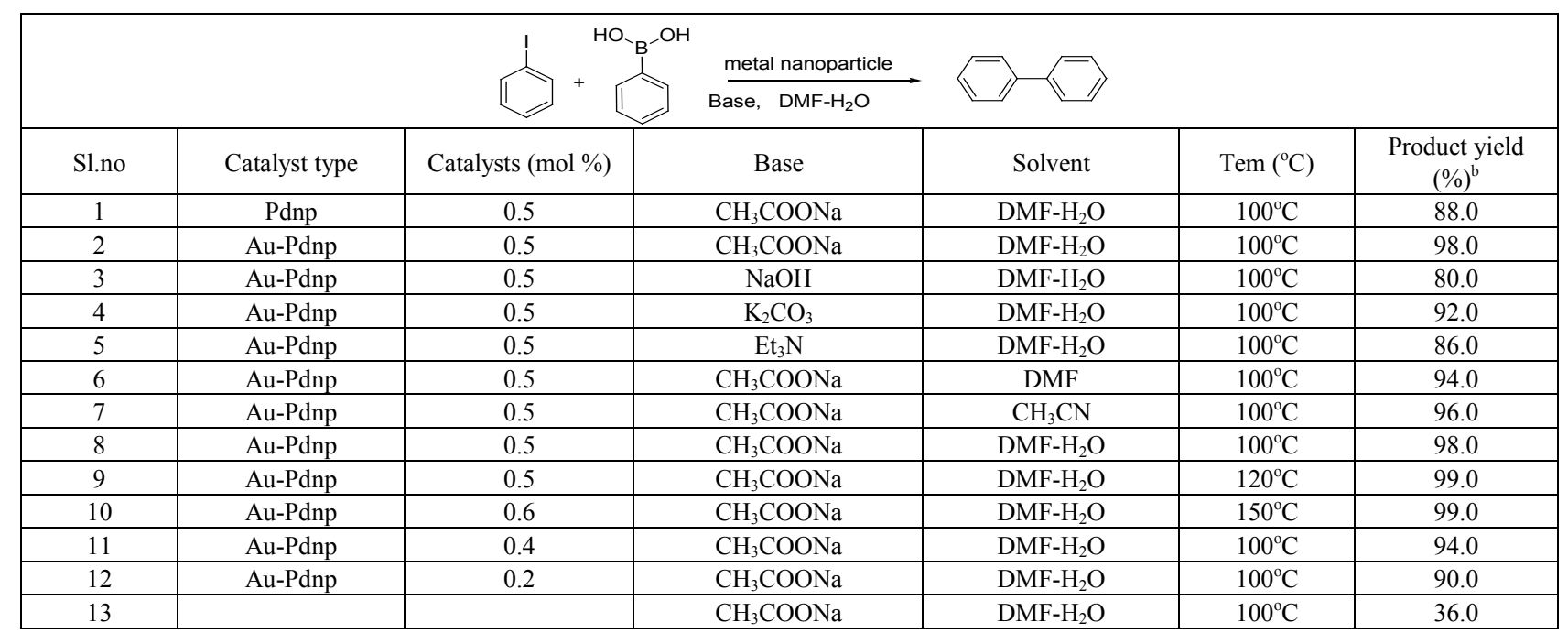

*Reaction conditions: Phenylboronic acid $(1.05 \mathrm{mmol})$, Iodobenzene $(1.00 \mathrm{mmol})$, Base $(3.00 \mathrm{mmol}), 12 \mathrm{hr}$; ${ }^{\mathrm{b}}$ Isolated yield. 


\subsection{The Study of Reaction Mechanism FT-IR Studies on the Suzuki Reaction Mechanism as KBr Pellets}

$\mathrm{KBr}$ enriched films of Sodium acetate (SA), Phenylboron ic acid (PA), SA + PA, CTAB-bimetallic nanoparticles, bnp $+\mathrm{PA}$, and bnp + SA + PA are prepared and dried in the oven for 30 minutes in order to investigate how the Phenylboronic acid interacts with the bnp surface. In addition, films of iodobenzene (I), SA + I, and bnp + SA + I are also prepared in order to investigate how the Iodobenzene interacts with the bnp surface. FTIR spectra of the films are recorded in the range of 4000 to $400 \mathrm{~cm}^{-1}$. Table 2 summarizes the important vibrational modes and the frequencies observed in Phenylboronic acid, Phenylboronic acid + sodium acetate, Phenylboronic acid + bimetallic nanoparticles, and Phenylboronic acid + sodium acetate + bimetallic nanoparticles.

Table 2. Frequencies of different vibration modes* in Phenylboronic acid (PA), PA + sodium acetate (SA), bimetallic nanoparticle (bnp) + PA, and bnp $+\mathrm{PA}+\mathrm{SA}$ at $25^{\circ} \mathrm{C}$

\begin{tabular}{|c|c|c|c|c|}
\hline \multirow{2}{*}{ Vibration Mode* } & \multicolumn{4}{|c|}{ Frequency in $\left(\mathrm{cm}^{-1}\right)$} \\
\cline { 2 - 5 } & PA & PA+SA & bnp+PA & bnp+PA+SA \\
\hline B-O Stretching & 1344 & 1346 & 1348 & 1383 \\
\hline B-C Stretching & 1085 & 1087 & 1069 & 1102 \\
\hline B-C Stretching & 1174 & 1180 & 1187 & 1179 \\
\hline $\begin{array}{c}\text { Out-of-plane } \\
\text { phenyl }\end{array}$ & & & & \\
\hline $\begin{array}{c}\text { ring deformation } \\
\text { Shoulder next to } \\
\text { out-of-plane } \\
\text { phenyl ring }\end{array}$ & 696 & 700 & 697 & 705 \\
\hline deformation & 650 & 579 & 652 & 581 \\
\hline
\end{tabular}

Figure 1 a-f shows the FT-IR spectra in the region of 4000 to $400 \mathrm{~cm}^{-1}$ obtained from dried films of bimetallic nanoparticles (bnp) (figure 1a), sodium acetate (SA) (figure 1b), Phenylboronic acid (PA) (figure 1c), SA + PA (figure 1d), PA + bnp (figure 1e), and bnp + PA + SA (figure 1f). Band assignments for PA are obtained from a previous infrared spectral study in the literature[50]. It can be seen that the B-O stretching band shifts from $1383 \mathrm{~cm}^{-1}$ in the presence of PA to $1344 \mathrm{~cm}^{-1}$ when the nanoparticles are added to PA + SA in solution before drying. In the spectra of $\mathrm{PA}+\mathrm{SA}$ without the presence of bimetallic nanoparticles, it is observed that the B-O stretching band occurs at $1346 \mathrm{~cm}^{-1}$, and this suggests that deprotonation does not occur in the film due to the loss of water upon drying and thus does not result in a shift in the B-O stretching mode. The large shift in the B-O stretching frequency that occurs when the bimetallic nanoparticles are added to PA and SA prior to evaporation of the solution is an important indication that the Phenylboron ate anion (formed from PA due to the presence of SA) binds to the nanoparticle surface through the B-O-group. In the case of the bimetallic nanoparticles $+\mathrm{PA}$, it is observed that the $\mathrm{B}-\mathrm{O}$ stretching mode occurs at $1348 \mathrm{~cm}^{-1}$, suggesting that the addition of PA does not result in the binding to the Pd-bimetallic nanoparticle surface. This provides additional evidence that the Phenylboronic acid must be in the form of Phenylboronate anion in the presence of sodium acetate in order to bind to the palladium nanoparticle surface.

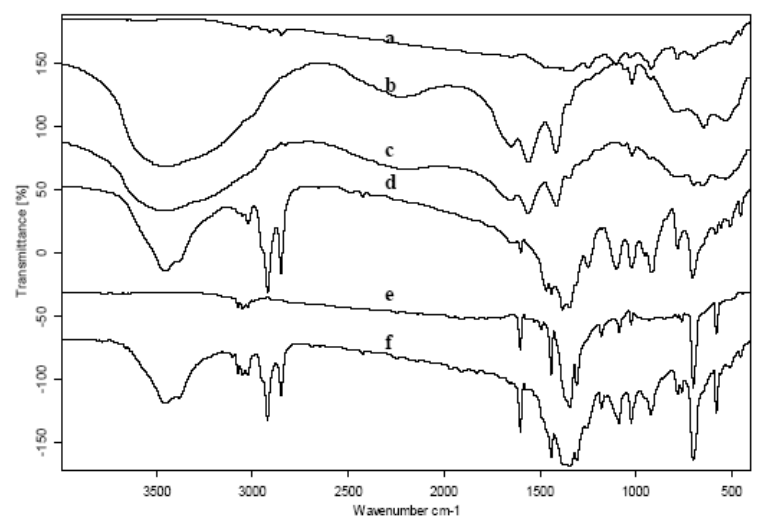

Figure 1. (a-f) a-f FTIR spectra of Phenylboronic acid (PA), Sodium acetate (SA), SA + PA, bimetallic nanoparticles (bnp), bnp + PA, and bnp + $\mathrm{SA}+\mathrm{PA}$ in 4000 to $400 \mathrm{~cm}^{-1}$ region (a) bnp, (b) SA, (c) PA, (d) SA + PA, (e), $\mathrm{PA}+$ bnp, (f) bnp $+\mathrm{SA}+\mathrm{PA}$ at $25^{\circ} \mathrm{C}$

An interesting question involves determining how the boronate group $\left(\mathrm{BO}^{2-}\right)$ of phenylboronate anion binds to the nanoparticle surface and Figure 2 illustrates two possible binding modes. The phenylboronate anion can bind to the bimetallic nanoparticle surface through a single bond between its B-O-group and the bnp atom (terminal binding) or through both of its B-O- groups to two bnp atoms (bridged binding). Terminal binding would result in bands existing in both the $1346 \mathrm{~cm}^{-1}$ and the $1383 \mathrm{~cm}^{-1}$ regions since one B-Ogroup would be bound to the bimetallic nanoparticles while the other B-O- group would be free. The blue shift in the B-O stretching mode that occurs when the nanoparticles are exposed to PA + SA is relatively large $\left(\sim 37 \mathrm{~cm}^{-1}\right)$. Since there is no band in the $1346 \mathrm{~cm}^{-1}$ region and only one shifted band is observed at $1383 \mathrm{~cm}^{-1}$, the phenylboronate anion most likely binds through a bridge involving two B-O-bnp bonds.

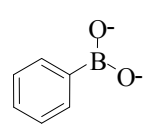

Phenylboronate Anion

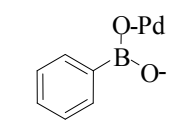

Terminal Binding of Phenylboronate Anion with single Pd-O-B group



Terminal Binding of Phenylboronate Anion with two Pd-O-B groups
Figure 2. Illustration of phenylboronate anion and the two possibilities of binding to the Au-Pd nanoparticle surface which can occur. The binding can occur through one B-O- group or through both B-O-groups

\section{FTIR Studies on Films of Iodobenzene and the Pd-bnp Nanoparticles interaction}

Figure 3a-e show FTIR spectra in the region of 4000 to $400 \mathrm{~cm}^{-1}$ for the following conditions involving bnp (figure $3 a$ ), sodium acetate (SA) (figure $3 b$ ), iodobenzene (I) (figure $3 c$ ), I + SA (figure 3d), and bnp + I + SA (figure 3e). The band assignments for iodobenzene were obtained from infrared spectral studies conducted in the literature[51]. It can be seen that there are no shifts in the bands that are associated with characteristic vibrational modes ofiodobenz ene. There are no shifts in the four C-C stretching modes at $1551 \mathrm{~cm}^{-1}, 1499 \mathrm{~cm}^{-1}, 1472 \mathrm{~cm}^{-1}$, and $1398 \mathrm{~cm}^{-1}$. There are 
no shifts in the C-I stretching mode at $1059 \mathrm{~cm}^{-1}$ and the $\mathrm{C}-\mathrm{C}-\mathrm{H}$ in-plane bending mode at $1079 \mathrm{~cm}^{-1}$. There are also no shifts in the ring breathing mode at $898 \mathrm{~cm}^{-1}$ or in the two $\mathrm{CH}$ out-of-plane bending modes at $845 \mathrm{~cm}^{-1}$ and $731 \mathrm{~cm}^{-1}$. Finally, there is also no shift in the C-C-C nonplanar twist mode at $688 \mathrm{~cm}^{-1}$. Since there are no shifts in the bands associated with the characteristic vibrational modes of iodobenzene, it is concluded that iodobenzene does not have specific bonding to the surface of the bimetallic nanoparticl es.

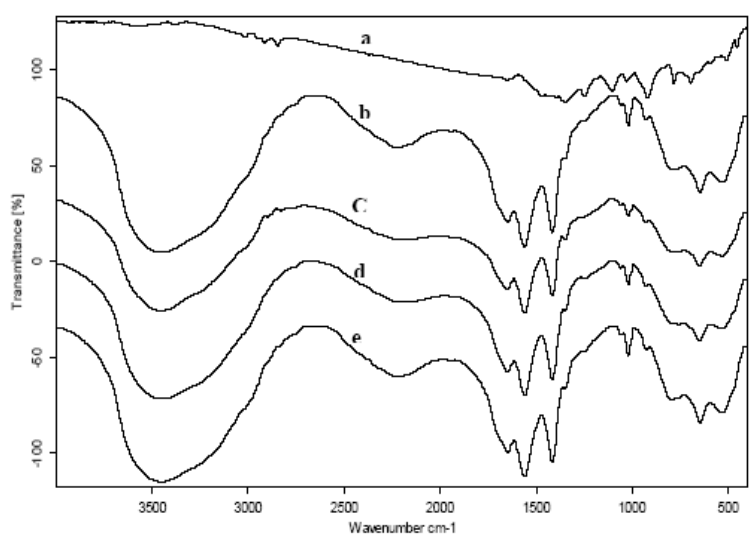

Figure 3. a-e FTIR spectra of Iodobenzene (I), Sodium acetate (SA), bimetallic nanoparticles (bnp), SA $+\mathrm{I}$, and bnp $+\mathrm{SA}+\mathrm{I}$ in 4000 to $400 \mathrm{~cm}^{-1}$ region (a) bnp, (b) SA, (c) I, (d) I $+\mathrm{SA}$, (e) $\mathrm{I}+\mathrm{SA}+$ bnp at $25^{\circ} \mathrm{C}$

\section{Conclusions}

In conclusion, we have synthesized a novel family of $\mathrm{Au}-\mathrm{Pd}$ core-shell bimetallic nanoparticles from the wet chemical method of metal-surfactant complexes has been reported our previously report. We have demonstrated that a colloidal solution of Au-Pd bimetallic nanoparticles is an efficient catalyst for Suzuki cross-coupling reactions in aqueous solution. The surface of palladium has a reasonably high surface area and pore volume making it an attractive host for the insertion of nanoparticles. The size of the bimetallic nanoparticle could be controlled within the CTAB. The UV-Vis, HRTEM, XRD, SEM-EDX, XPS and FTIR analyses all suggest the formation of a core-shell Au-Pd structure. The catalytic activity of the Suzuki coupling react ion has monitored by FTIR spectroscopy. The bimetallic nanoparticles were found to have high catalytic activity than mono metallic nanoparticle. The high catalytic activity of bimetallic nanoparticles is probably due to the sequential electronic effect between elements in a particle. More detailed investigations of nanoparticle structure effects on the catalytic activity and their applicability in other synthetic transformations are currently under investigation.

\section{ACKNOWLEDGMENTS}

The authors thank financial support to the Department from DST-FIST

\section{REFERENCES}

[1] Alain Roucoux, Jurgen Schulz, and Henri Patin, Chem. Rev. 102 (2002) 3757 - 3778.

[2] Peiwen Zheng, and Wangging Zhang. J. catal. 250 (2007) 324 -330 .

[3] L. N. Lewis, Chem. Rev. 93 (1993) 2693 - 2730.

[4] J. D. Aiken, Y. Lin, and R. G. Finke, J. Mol. Catal. A: Chem. 114 (1996) 29 - 51.

[5] H. Tsunoyama, H. Sakurai, Y. Negishi, and T. Tsukuda, J. Am. Chem. Soc. 127 (2005) 9374 - 9375.

[6] Yin Li, Edna Boone, and Mostafa A. El-Sayed, Langmuir. 18 (2002) 4921 - 4925.

[7] J. Dai, and M. L. Bruening, Nano Lett. 2 (2002) 497 - 501.

[8] W. Yoo, D. Hathcock, and M. A. El-Sayed, J. Phys. Chem. A. 106 (2002) 2049 - 2054.

[9] M. Nakaya, M. Kanehara, and T. Teranishi, Langmuir. 22 (2006) 3485 - 3487.

[10] X. Du, and N. Toshima, Chem. Lett. 35 (2006) 1254 - 1255.

[11] M. Chen, J. Kim, J. P. Liu, H. Fan, and S. Sun, J. Am. Chem. Soc. 128 (2006) 7132 - 7133.

[12] S. O. Keun, E. L. Kyung, S. H. Sung, H. C. Sun, K. Dongmin, and Y. Soon Hong, Biomacromolecules. 6 (2005) 1062 1067.

[13] Ajay Kumar Gupta, Stephen Wells, and Ieee Transactions On Nanobioscience, 3 (2004) 66 - 84.

[14] T. Teranishi, M. Nishida, and M. Kanehara, Chem. Lett. 34 (2005) 1004 - 1005.

[15] N. Watanabe, and N. Toshima, Bull. Chem. Soc. Jpn. 80 (2007) 208 - 214.

[16] R. P. Andres, T. Bein, M. Dorogi, S. Feng, J. J. Henderson, C. P. Kubiak, W. Mahoney, R. G. Osifchin, and R. Reifenberger. Science 272 (1996) 1323 - 1325.

[17] P. Galletto, P. F. Brevet, H. H. Girault, R. Antoine, and M. Broyer. J. Phys. Chem. B 103 (1999) 8706 - 8710.

[18] W. Baschong, and N. G. Wrigley, J. Electron Microsc. Tech. 14 (1990) 313 - 323

[19] W. J. Parak, T. Pellegrino, C. M. Micheel, D. Gerion, S. C. Williams, and A. P. Alivisatos, Nano Lett. 3 (2003) 33 - 36.

[20] Shiho Tokonami, Nobuyasu Morita, Kanako Takasaki, and Naoki Toshima J. Phys. Chem. C. (2010, in press)

[21] Karl J. J. Mayrhofer, Katrin Hartl, Viktorija Juhart, and Matthias Arenz J. Am. Chem. Soc., 2009, 131 (45), 16348-16349.

[22] N. Aihara, K. Torigoe, and K. Esumi, Langmuir 14 (1998) 4945 - 4949.

[23] P. Laura, V. Alberto, C. Claudio, and S. Paolo, Topics in 
Catal. 44 (2007) 319 - 335.

[24] J.K. Edwards, B.E. Selsone, P. Landon, A.F. Carley, A. Herzing, C.J. Kiely, and G.J. Hutchings, J. Catal. 236 (2005) $69-79$.

[25] M.O. Nutt, J.B. Hughes, and M.S. Wong, Environ, Sci. Technol. 39 (2005) 1346 - 1353.

[26] Maureen R. Regan, and Ipsita A. Banerjee, Scripta Materialia. 54 (2006) 909 - 914.

[27] N. Miyaura, T. Yanagi, and A. Suzuki, Synth. Commun. 11 (1981) 513 - 519

[28] N. Miyaura, and A. Suzuki, Chem. Rev. 95 (1995) 2457 2483.

[29] A. Suzuki, J. Organomet. Chem. 576 (1999) 147 - 148.

[30] N. Miyaura, Top. Curr. Chem. 219 (2002) 11 - 59.

[31] N. Kataoka, Q. Shelby, J.P. Stambuli, and J.F. Hartwig, J. Org. Chem. 67 (2002) 5553 - 5566.

[32] M. Miura, Angew. Chem., Int. Ed. 43 (2004) 2201 - 2203.

[33] K.W. Anderson, and S.L. Buchwald, Angew. Chem., Int. Ed. 44 (2005) 6173 - 6177.

[34] K. Sarkar,M. Nandi, M. Islam,M.Mubarak, and A. Bhaumik, Appl. Catal. A: Gen. 352 (2009) 81 - 86.

[35] M. Joshaghani, E. Faramarzi, E. Rafiee, M. Daryanavard, J. Xiao, and C. Baillie, J. Mol. Catal. A: Chem. 259 (2006) 35 40

[36] T.E. Barder, J. Am. Chem. Soc. 128 (2006) 898 - 904.

[37] P. Han, H.M. Zhang, X.P. Qiu, X.L. Ji, and L.X. Gao, J. Mol. Catal. A: Chem. 295 (2008) 57 - 67.
[38] C. J. Li, Chem. Rev. 105 (2005) 3095 - 3166.

[39] E. Alacid, and C. Najera, Org. Lett. 10 (2008) 5011 - 5014.

[40] . Ma, X. L. Cui, L. Y. Gao, and Y. J. Wu, Inorg. Chem. Commun. 10 (2007) 762 - 766.

[41] J. Santhanalakshmi, and P. Venkatesan. J. nanopart. Res. 13, (2011), $479-490$

[42] J. A. Creighton, and D. G. Eadon, J. Chem. Soc., Faraday Trans. 87 (1991) 3881 - 3891.

[43] N. Toshima, and T. Yonezawa, New J. Chem. 22 (1998) 1179 -1201 .

[44] N. Toshima, M. Harada, Y. Yamazaki, and K. Asakura, J. Phys. Chem. 96 (1992) 9927 - 9933.

[45] J. A. Creighton, and D. G Eadon, J. Chem. Soc. Faraday Trans., 87 (1991) 3881 - 3891.

[46] T. E. Furtak, and J. Kester, Phys. Rev. Lett. 45 (1980) 1652 1655.

[47] C. J. Mathews, P. J. Smith, and T. Welton, Chem. Commun. (2000) $1249-1250$.

[48] R. Rajagopal, D. V. Jarikote, and K. V. Srinivasan, Chem. Commun. (2002) 616 - 617.

[49] J. D. Revell, and A. Ganesan. Org. Lett. 4 (2002) 3071 - 3073.

[50] R. Narayanan, R. J. Lipert, and M. D. Porter, Anal. Chem. 80 (2008) 2265 - 2271.

[51] D. Syomin, and B. E. Koel. Surf. Sci., 490 (2001) 265 - 273. 